\title{
Different Sizes of Windows for Energy Spectrum of a Photon Counting X-ray Imaging System
}

\author{
${ }^{1}$ Muhammad Masood \\ Sarfaraz and ${ }^{2}$ Aamir Khan \\ COMSATS Institute of IT, \\ WahCantt. Pakistan.
}

\author{
Farah Batool \\ GC University Faisalabad, \\ Pakistan
}

\author{
Adeel Akram, Farman \\ Ullah \\ COMSATS Institute of IT, \\ WahCantt. Pakistan.
}

\begin{abstract}
In relation to the radiation imaging sensor Timepix, the energy spectrum generated from the sensor data has been studied and for this, different sizes of windows have been selected in order to observe their effect on energy spectrum generation. It was found that removing the charge sharing effect significantly improves the means of discovering the centre peak. Additionally, it has been observed that a $4 \times 4$ window is best suited with regards to energy spectrum generation. Different fluorescent materials were used as samples in order to generate test images and the analysis was conducted using MATLAB.
\end{abstract}

Keywords: Radiation Imaging sensor, Timepix, Energy Spectrum.

\section{INTRODUCTION}

Timepix is an excellent chip and can be used as a medical imaging sensor as it is still too small and only has the ability to handle low flux rates. At this point, more research is required to overcome these problems. The aim is to attempt to discover some better solutions in order to enhance the features of the present Timepix. In order to achieve, analysis has been performed using different metals. The tests and the analysis have been conducted using MATLAB. The key point is to discover a method which will provide the best possible results. It is hoped that the results thus obtained can then be integrated into a future version of the Timepix chip and thus produce improved results.

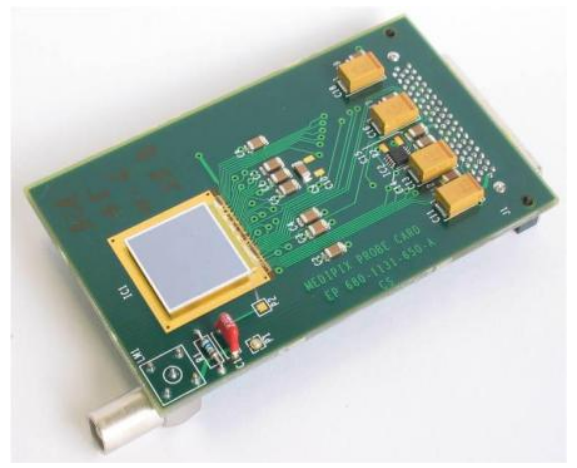

FiG.1: TIMEPIX CHIP[1]

\section{THEORY AND SYSTEM DESIGN}

Measurement setup consists of a hybrid silicon pixel device, the Timepix, is used. This device is the combination of two chips which are connected together by a bump-bonding method. One chip is a pixelated $300 \mu \mathrm{m}$ thick silicon detector chip and other is a bottom read-out chip with matrix dimensions of $256 \times 256$. This chip was developed at CERN by the MEDIPIX collaboration [2]. In this setup, fluorescence materials are placed in front of an $\mathrm{x}$-ray radiation source to create fluorescence radiation and a detector is placed behind the source at a certain angle. A schematic of this setup is shown in figure 2 while the actual setup is shown in figure 3 below.

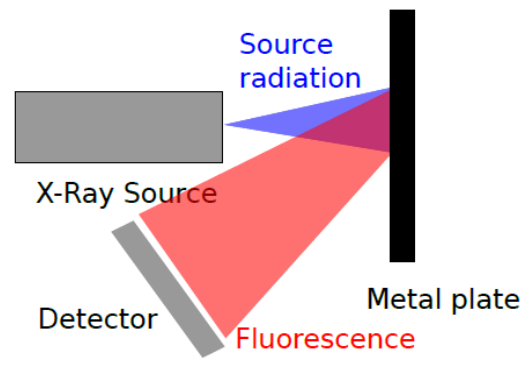

Fig. 2: Measurement Setup[3]

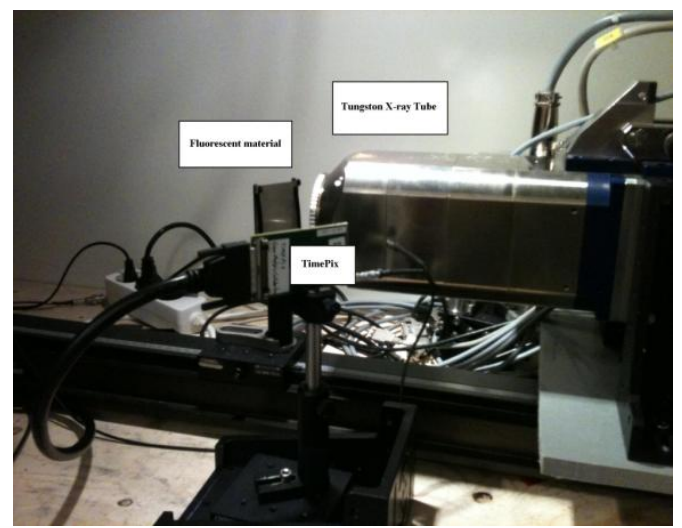

Fig.3: Measurement Setup in the lab

Pixel cell: Although the architecture of MEDIPIX resembles that of the Timepix cell there are three features in the cell that differentiate the two. Figure 4shows the schematics of the Timepix pixel cell. 


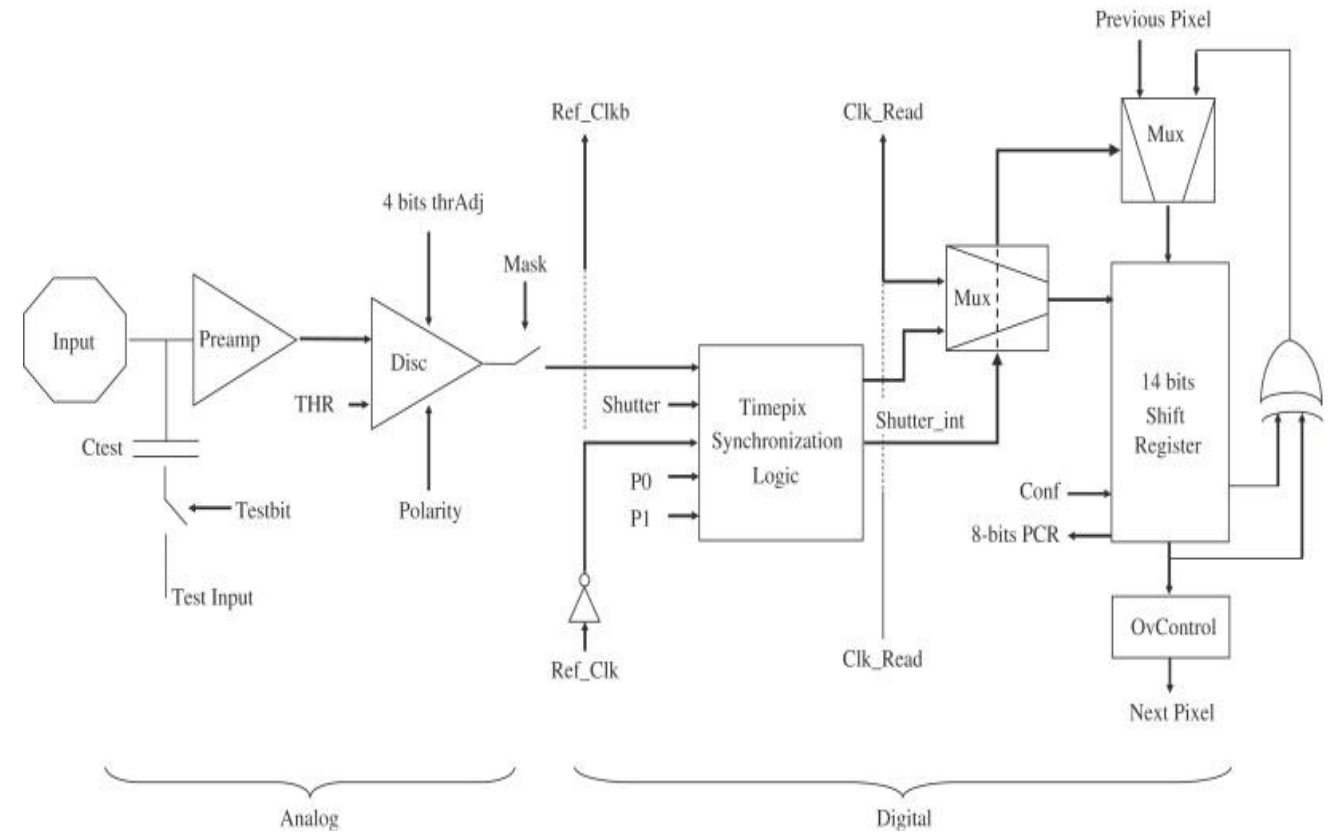

Fig.4: Timepix pixel cell schematic[4]

Charge sharing-Cluster:The charge collected by each pixel in the cluster can be measured using the Timepix device. The charge collection speed depends on the applied bias voltage and the cluster size (number of pixels in the cluster) also depends on that voltage. The number of pixels in one cluster is called the cluster size. If measurements are performed without removing the cluster then the resulting spectrum is distorted. Thus a calibration of the Timepix is required in order to produce a satisfactory spectrum [5].If the computation of a cluster volume is conducted without any pixel calibration then the resulting spectrum is distorted.

Pixelman: The Institute of Experimental and Applied Physics (IEAP) at the Czech Technical University (CTU) in Prague have developed software for the MEDIPIX2 acquisition control and have named it Pixelman [9]. This software supports all the MEDIPIX family devices including the MEDIPIX 2, 2.1, MXR, MEDIPIX2 MXR Quad, Timepix and Timepix Quad. All of this is achieved based on their internal flexibility.In order for these to operate for different options, it is necessary to use custom made plug-ins. Threshold equalization is used in order to compensate for the pixel to pixel threshold variations.

The adjustment code is then selected for each pixel in order to make its threshold as near as possible to the average of the threshold distribution mean values. In other words it can be stated that threshold equalization is a procedure in which THL/THH adjustment is performed so as to make all the thresholds as homogenous as possible.Figure 5 below shows the threshold equalization of the TIMEPIX detector.

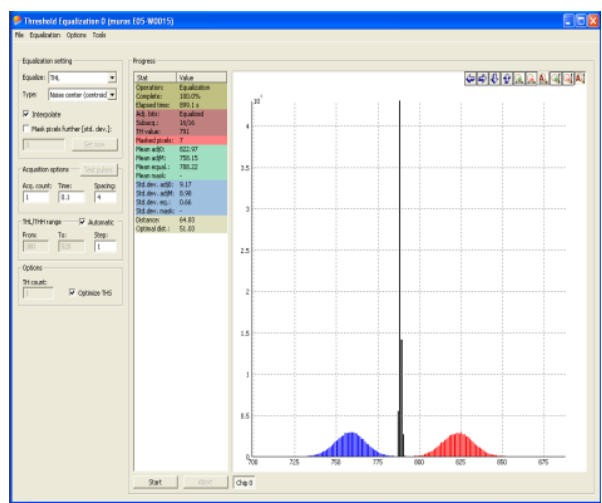

Fig.5: Threshold Equalization of Timepix Detector

Time over Threshold calibration:Before it is possible to make any measurements of metals, the TIMEPIX detector must be calibrated.

For this purpose the TIMEPIX will be used in Time-overThreshold (TOT) mode in which a single pixel is used for the calibration. The time -over-threshold will calibrate the energy.The calibration technique used in this case is different to that of the technique given in the TIMEPIX home page [5]. In this case, the maximum peaks of the fluorescence materials are used and these are then used for the calibration. A Gaussian curve fitting method is used in order to determine the maximum peak of the fluorescence materials. Figure 6 below displays the TOT calibration curve 


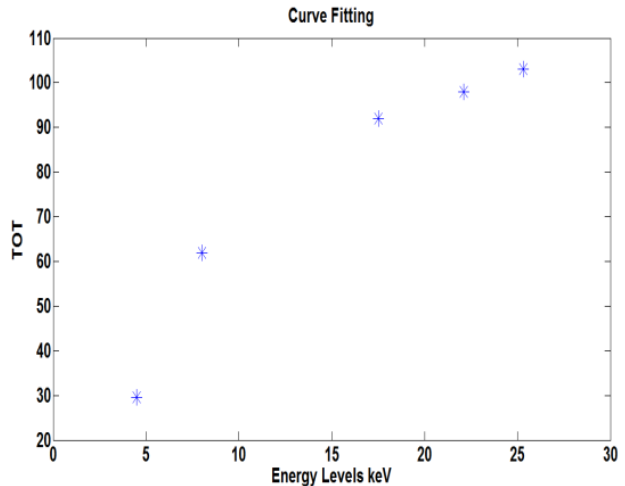

Fig.6:TOT Calibration measuerment

The mathematical equation below is used in order to verify the calibration

$$
\begin{gathered}
y=a x+b-\frac{c}{x-t} \\
y(x-t)=a x(x-t)+b(x-t)-c \\
x=\frac{-(b-a t-y) \pm \sqrt{\left.b^{2}+a^{2} t^{2}+y^{2}-2 b a t-2 b y+2 a t y+4 a b t-4 a t y-4 a c\right)}}{2 a}
\end{gathered}
$$

and finally the calibration.

$$
x=\frac{t a+y-b+\sqrt{(b+t a-y)^{2}+4 a c}}{2 a}
$$

Fluorescence-In medical science, different fluorescence materials are used in order to detect and visualize the structure and process of biological samples. The reason why these are used is because the fluorescence molecule has extraordinary selectivity and sensitivity. How is it possible for fluorescence material to emit different colors of light?Some molecules are capable of becoming excited by the absorption of light energy which results in a higher energy state which is called the excited state. This excitation cannot be sustained for any length of time and the result of this is the emission of light energy. This process is called fluorescence [6]. Fluorescence means to emit light and this process from absorption to emission is shown in Figure 7.

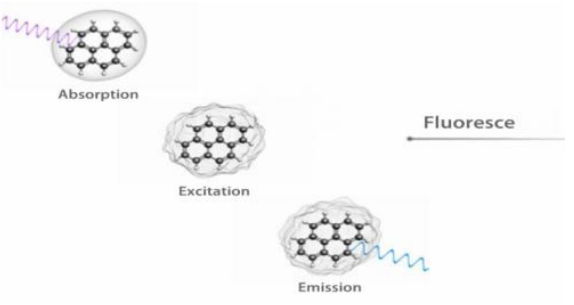

Fig.7:Flourescense Procedure[6]

Fluorescence has many applications including dyes, biological detectors, chemical sensor and the very common fluorescence lamps.Materials used as fluorescence are given in the table 1 below.
Table 1: Materials used as fluorescence

\begin{tabular}{|l|l|l|l|}
\hline Sr.No & Materials & Energy & TOT \\
\hline $\mathbf{1}$ & Copper(cu) & $8.0 \mathrm{KeV}$ & 62 \\
\hline $\mathbf{2}$ & Molybdenum(mo) & $17.5 \mathrm{KeV}$ & 92 \\
\hline $\mathbf{3}$ & Silver(Ag) & $22.1 \mathrm{KeV}$ & 98 \\
\hline $\mathbf{4}$ & Tin(Sn) & $25.3 \mathrm{KeV}$ & 103 \\
\hline
\end{tabular}

Some characteristic of the materials are described in figures 2.7 and 2.8 below. As disused above copper, molybdenum, silver and tin are used as X-ray fluorescence. X-ray fluorescence is a processes related to $\mathrm{x}$-ray absorption. The energy of generated X-ray photon typically corresponds to the binding energy of K-shell electron. The energy of fluorescent photons increases with atomic number in figures 8 and 9 . Fluorescent X-rays are emitted in any direction and can be absorbed again. X-ray fluorescence process is clearer in section above. 


\section{$2,18,8,1 \quad 2,8,18,18,1$}

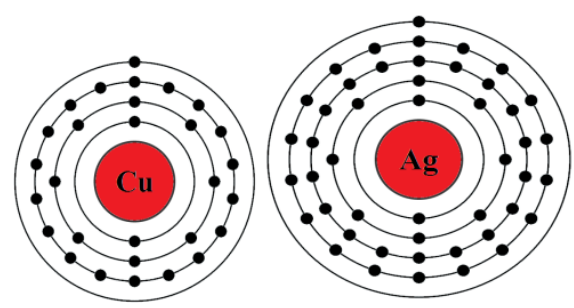

Fig.8:Copper Fig.9:Silver

It is known that the Timepix provides the energy directly from the pixels. In this case, use is being made of low flux and the photons are spread out in the image. One photon can generate the signals and multiple pixels by selecting different windows. In this way we can utilize the large pixels because this chip is so sensitive to signals received from several pixels. It is thus possible to achieve better energy spectrums using different windows.In an attempt to discover a better energy spectrum for the different materials, all the pixels in the image must firstly be taken. In this case there is no need to delete the single hit or the charge spread over two pixels and then to plot against the energy scale.Figure 10 shows a small image with all pixels.

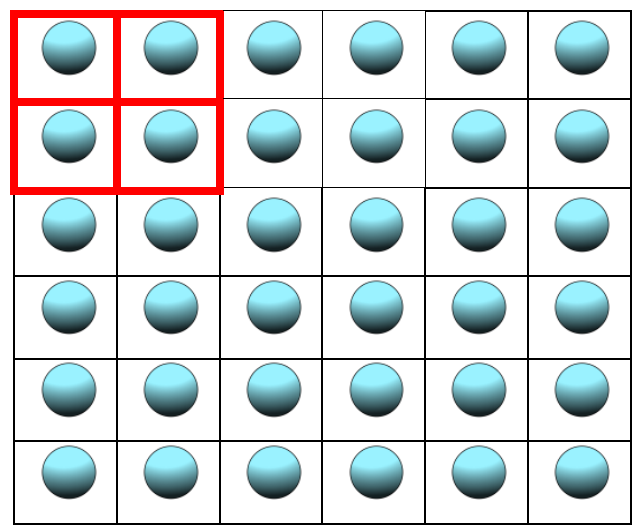

Fig.10: taking whole pixels of the whole image without deleting any single hit or double hit.
Different windows method to find out better energy spectrum: There are different choices available for the different windows methods in an attempt to discover a better energy spectrum. A better energy spectrum means that there will be very sharp peaks and the sharper the peak the better the sensor will perform. Plot all the windows in one plot and make a comparison to determine which window size is better, regarding different energies, in achieving a better energy spectrum. The responses of the sensor and the manner in which it works are shown and this has been conducted without deleting any single or double hits.This has been conducted in order to determine which window size is best in achieving a better energy spectrum. Images have been processed into MATLAB. In this case it firstly selects a $1 \times 1$ window, then a $2 \times 2$ window and continues with a $3 \times 3$ and a $4 \times 4$ window and then converts these times over threshold (TOT) values into energy by using the function "tot_to_energy".

The reason for performing the addition as the first task is because the energy dependence of TOT is not linear and this is particularly true in relation to lower energies. Thus the TOT values are firstly added together and are then converted into energy. After the conversion of the TOT to energy, higher energies are obtained and the pixels are then summed together. If the energy dependence of the TOT is linear then it is possible to go directly to the summation step. The next step is to sum of all the energy values. The summed value is then again converted back to time over threshold by using the function "energy_to_tot".

Functions that convert time over threshold values into energy (tot_to_energy) and or convert energy values into time over threshold values (energy_to_tot) were developed in MATLAB for this purpose. It is important to note that the conversion of values from TOT to energy and then back to TOT is necessary. For some reason, a certain amount of noise can exist which may cause the production of errors in the results and in order to rectify this problem, the inter-conversion of TOT and energy values is performed.Figure 11 shows a block diagram and the box diagrams in figures 12, 13 and 14 show how the different windows are selected and provides a clear display regarding the selection of pixels in a window.

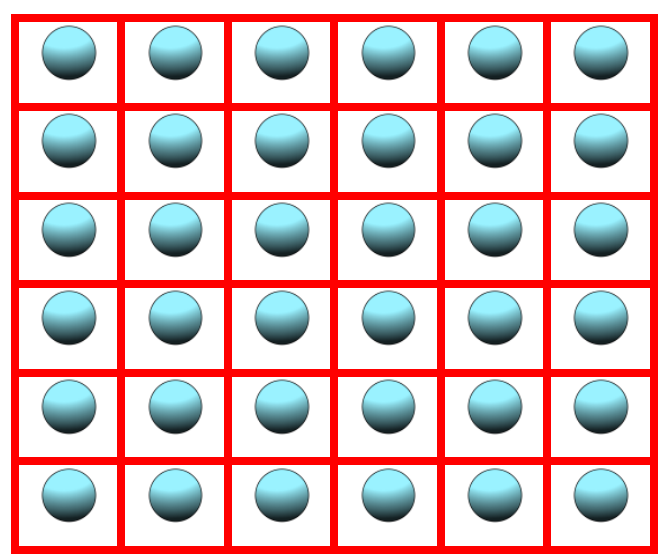

Fig.12: $2 \times 2$ pixel window the image. 


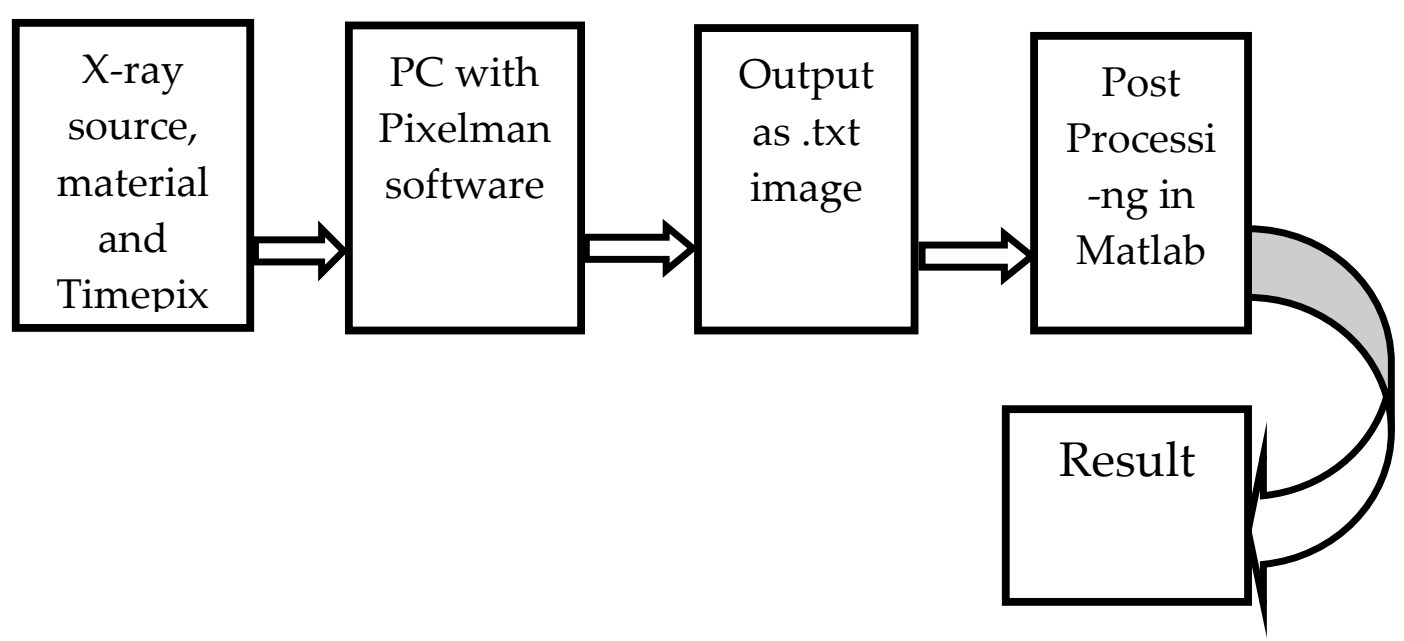

Fig.11: Basic design Model
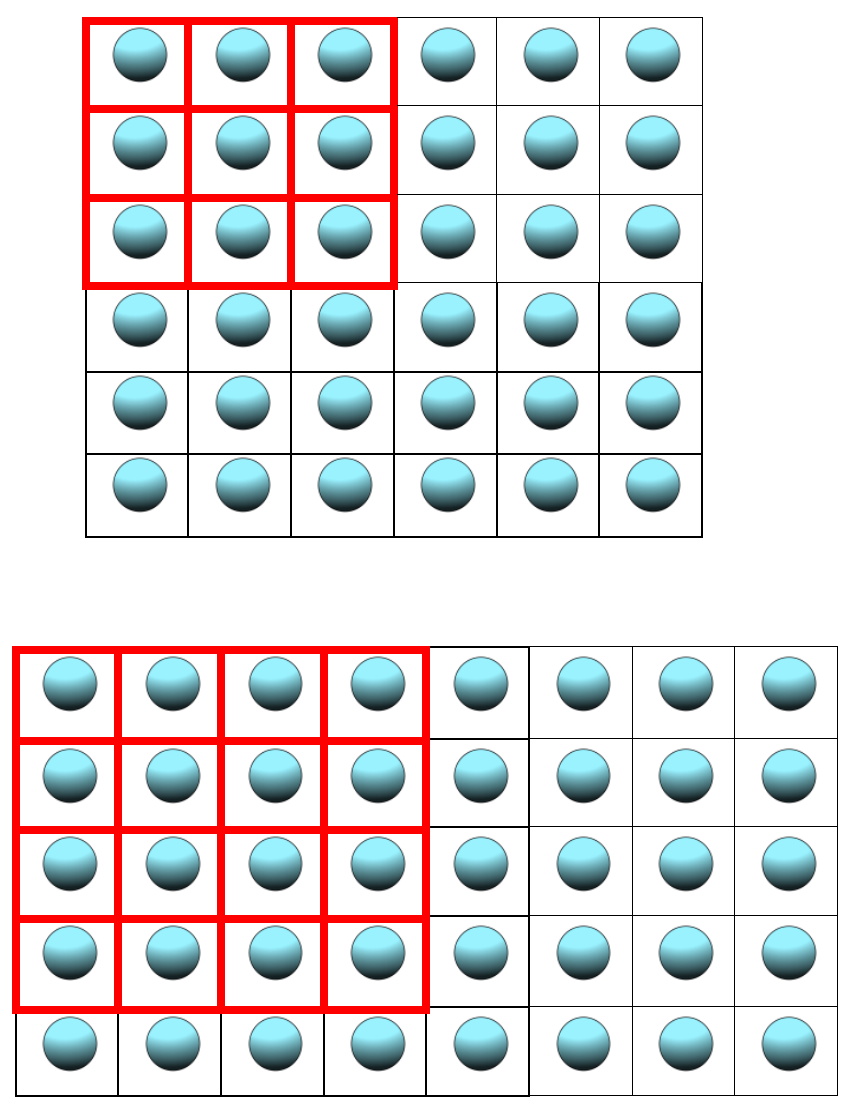

Fig. 13: $3 \times 3$ pixel window of the image.

Fig.14: $4 \times 4$ pixel window of the image.

\section{RESULTS}

In order to achieve a better energy spectrum for the Molybdenum, Copper, Silver and Tin, different sizes of windows have been used. Metals are used in order to obtain monochromatic radiation. The requirement in this case is to analyze which metal has the better energy spectrum in relation to different energy levels. In principal, a comparison is being made in relation to the different windows sizes and the attempt is to discover the window which offers the sharpest peak with the minimum noise for a maximum number of hits against a given energy level. As discussed above, the sharper the peak the better will be the performance of the sensor. A comparison of the different windows in the Copper energy spectrum shows that there is no improvement.

Firstly, the energy of copper is very low as there is only minor charge sharing and thus there is only a limited option to add values. In addition the THL values are low and thus no pulse will be detected and values are unable to be added. This could be stated as there being less charge clouds in the sensor and thus, for the given reasons, there is no improvement in the copper. The results for the copper energy spectrum after comparing different windows is shown in figure 15 .

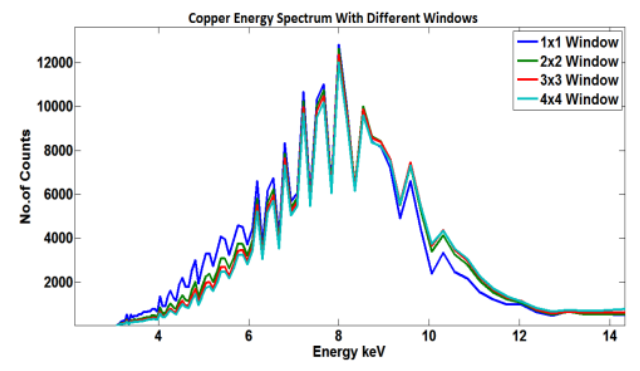

Fig.15: Energy Spectrum of Copper With Different Windows 
However, for the cases involving molybdenum, silver and tin there will be different scenarios when comparisons are made for different window sizes. The energy of these will be high as will the charge sharing in the sensor and thus the values can be added. The higher energy values for these cases means that they are above the threshold and thus it is possible to add the pixel values. The energy spectrums of molybdenum, silver and tin were observed at different energies. In all cases the energy spectrum in the $4 \times 4$ window proved to be the best as it showed both the sharpest peak and the lowest noise. The results for the molybdenum, silver and tin energy spectrums after comparing different window sizes are shown in figures 16,17 and 18 .

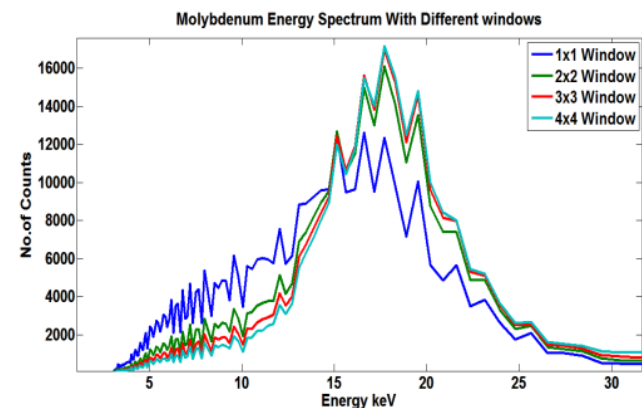

Fig.16: Energy Spectrum of Molybdenum With Different Windows

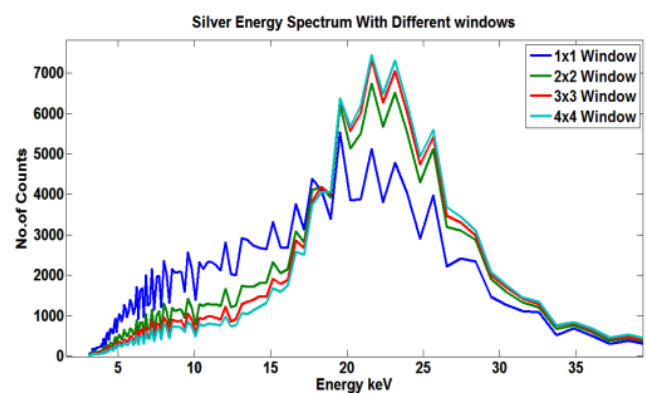

Fig. 17: Energy Spectrum of Sliver With Different Windows

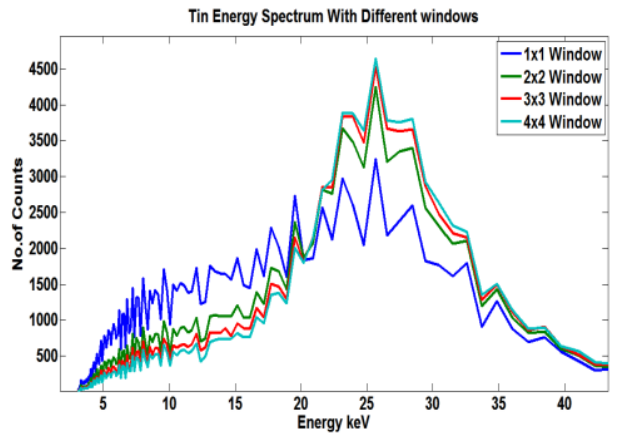

Fig.18: Energy Spectrum of Tin With Different Windows

\section{RESULTS}

System was successfully calibrated so that the response of the system could be observed for different metals at different energy levels. Furthermore it has been found that the windowing method for energy spectrum calculation has revealed that the best results are obtained for all metals using a $4 \mathrm{x} 4$ window. $2 \mathrm{X} 2$ window is enough for the energy range (8 $\mathrm{KeV}$ to $25 \mathrm{KeV}$ ) of copper, molybdenum, silver and tin but there is some chance it doesn't get all pixels. Than by increasing $3 \mathrm{X} 3$ or $4 \mathrm{X} 4$ is big chance to whole hits inside the window. It means that it captures large charge clouds within one pixel. And larger pixel will statistically capture a larger part of the charge clouds that corresponds to photons. By doing this charge sharing will be reduced. The analysis and the results thus generated during this thesis work shows that the charge summing strategy implemented in MEDIPIX3 will successful for applications in this energy ranges.

\section{REFERENCES}

[1] Victor Gutierrez Diez,'Measurements of time pix detectors performance', Universidad Complutense de Madrid, September 2010.

[2] http://aladdin.utef.cvut.cz/ofat/Methods/TimePixCalibrat ion/index.html

[3] Erik Fröjdh,'X-ray absorption and charge transport in a pixellatedCdTe detector with single photon processing read out', Mid Sweden University , Holmgatan 10,851 70 Sundsvall, Sweden .

[4] X. Llopart,' Timepix a 65K programmable pixel readout chip for arrival time, energy and/or photon counting measurements', Volume 581, Issues 1-2,21 October 2007 , pages $485-494$. VCL 2007-Proceedings of the $11^{\text {th }}$ International Vienna Conference on Instrumentation.

[5] Medipix in IEAP, available at: http://aladdin.utef.cvut.cz/ofat/Methods/TimePixCalibrat ion/index.htm\#Clusters

[6] Fluorescence Tutorials, available at http://www.invitrogen.com/site/us/en/home/support/Tuto rials.html

[7] Glenn F. Knoll, 'Radiation Detection and Measurement', ISBN 0-471-07338-5

[8] Syed Naeem Ahmed, 'Physics \& Engineering of Radiation Detection by', ISBN -10:0-12-045581-1

[9] Muhammad MasoodSarfaraz, "An Investigation of How Pixel Size Affects the Energy Resolution of a Photon Counting X-ray Imaging System”, MS Thesis 2011 